\title{
Arborescences
}

Revue d'études françaises

\section{Quand le narrateur boit(e)... (Réflexions sur le narrateur non fiable et/ou indigne de confiance)}

\section{Frank Wagner}

Numéro 6, septembre 2016

Polyphonies : voix et valeurs du discours littéraire

URI : https://id.erudit.org/iderudit/1037508ar

DOI : https://doi.org/10.7202/1037508ar

Aller au sommaire du numéro

Éditeur(s)

Département d'études françaises, Université de Toronto

ISSN

1925-5357 (numérique)

Découvrir la revue

Citer cet article

Wagner, F. (2016). Quand le narrateur boit(e)... (Réflexions sur le narrateur non fiable et/ou indigne de confiance). Arborescences, (6), 148-175.

https://doi.org/10.7202/1037508ar

\section{Résumé de l'article}

Cet article consiste en une tentative de mise au point sur la question du narrateur non fiable et/ou indigne de confiance - "procédé » textuel susceptible de nous aider à formaliser indirectement la problématique de la polyphonie du texte littéraire. Y est tout d'abord esquissée une forme d'état des lieux métathéorique des thèses des tenants de l'approche "rhétorique " (Booth), de la narratologie structurale (Lintvelt, Jouve) et du cognitivisme (Fludernik, Nünning), dans l'espoir de clarifier les critères nécessaires à l'établissement du défaut de fiabilité de l'instance narrative. Sont ensuite évoquées, dans une perspective plus critique, quelques typologies poétologiques (Mercier et Fortier, Langevin) permettant de repérer les variantes textuelles du " procédé " ainsi que la diversité de ses fonctions et de ses effets. On espère ainsi qu'à l'issue de ce parcours, les tenants et aboutissants esthétiques autant qu'épistémologiques du récit à narrateur non fiable et/ou indigne de confiance auront pu être quelque peu clarifiés - de même, par la bande, que la question de la polyphonie du texte littéraire.
Tous droits réservés ( D Département d'études françaises, Université de Toronto, 2016
Ce document est protégé par la loi sur le droit d'auteur. L'utilisation des services d’Érudit (y compris la reproduction) est assujettie à sa politique d'utilisation que vous pouvez consulter en ligne.

https://apropos.erudit.org/fr/usagers/politique-dutilisation/ 


\section{SOMMAIRE}

Francis Langevin et Raphaël Baroni.

01 University of British Columbia et Université de Lausanne Introduction

Alain Rabatel. Université de Lyon 1

13 L'énonciation problématisante : en dialogue avec

Le Royaume d'Emmanuel Carrère

Émilie Goin. Université du Luxembourg

39 Analyse d'un discours d'action collective mis en récit.

L'Anarchie dans Les Cloches de Bâle d'Aragon

David Bélanger. Université du Québec à Montréal

54 En contre-jour : la représentation évanescente de l'écrivain dans le roman québécois contemporain

Raphaël Baroni. Université de Lausanne

72 Comment débusquer la voix d'un auteur dans sa fiction?

Une étude de quelques provocations de Michel Houellebecq

Liesbeth Korthals Altes. Rijksuniversiteit Groningen

94 Actes de cadrage, narratologie et herméneutique - à propos de I'indétermination énonciative dans Sujet Angot de Christine Angot

121

Marion Sauvaire et Érick Falardeau. Université Laval

Susciter le moment critique. De l'investissement de ressources axiologiques

Frank Wagner. Université Rennes 2

148 Quand le narrateur boit(e)... (Réflexions sur le narrateur non fiable et/ou indigne de confiance) 


\title{
Quand le narrateur boit(e)... (Réflexions sur le narrateur non fiable et/ou indigne de confiance)
}

\author{
Frank Wagner. Université Rennes 2
}

\section{Résumé}

Cet article consiste en une tentative de mise au point sur la question du narrateur non fiable etlou indigne de confiance - "procédé" textuel susceptible de nous aider à formaliser indirectement la problématique de la polyphonie du texte littéraire. Y est tout d'abord esquissée une forme d'état des lieux métathéorique des thèses des tenants de l'approche "rhétorique" (Booth), de la narratologie structurale (Lintvelt, Jouve) et du cognitivisme (Fludernik, Nünning), dans l'espoir de clarifier les critères nécessaires à l'établissement du défaut de fiabilité de l'instance narrative. Sont ensuite évoquées, dans une perspective plus critique, quelques typologies poétologiques (Mercier et Fortier, Langevin) permettant de repérer les variantes textuelles $d u$ "procédé" ainsi que la diversité de ses fonctions et de ses effets. On espère ainsi qu'à l'issue de ce parcours, les tenants et aboutissants esthétiques autant qu'épistémologiques du récit à narrateur non fiable et/ou indigne de confiance auront pu être quelque peu clarifiés - de même, par la bande, que la question de la polyphonie du texte littéraire.

C'est trop de Gaulois, ils sont ivrognes ! et trop de Crétois, ils sont menteurs! (Flaubert, Salammbô)

Commençons par une devinette : "Quel est le point commun entre Jeanne d'Arc et la plupart des narratologues ?... " À l'intention de celles et ceux qui donnent leur langue au chat, s'il y en a, voici la réponse : "Ils entendent des voix ». Dans une perspective narratologique, le texte littéraire peut en effet être considéré comme un carrefour de voix, puisque s'y font entendre à la fois celles des personnages, celle de l'instance qui les médiatise (le narrateur), et celle de l'instance surplombante en charge notamment de la construction de la figure narratoriale (le scripteur, l'auteur impliqué, l'auteur abstrait, etc. - selon les diverses terminologies en vigueur).

Je dois l'idée de cet article, ainsi que la connaissance de plusieurs des sources qui y sont mentionnées, à Anaïs Oléron, qui réalise actuellement, à l'université de Reims, un doctorat de littérature française, intitulé Le narrateur non fiable en question(s), parcours textuels et liberté du lecteur, sous la direction conjointe de Vincent Jouve et de moi-même. En espérant n'avoir pas trop empiété sur ses brisées, j'adresse ici mes sincères remerciements à Anaïs Oléron, dont les travaux m’ont vivement incité à tenter de formaliser une problématique que je n'avais jusqu'à ce jour fait qu'effleurer, dans le cadre de mes recherches sur la voix narrative.

\section{Arborescences}


Aussi consensuelle puisse-t-elle paraître, cette caractérisation du récit de fiction ne fait pourtant pas l'unanimité chez les théoriciens. Si l'attribution d'une voix aux personnages ne suscite pas à ma connaissance de controverses particulières, il en va différemment de l'hypothèse de la voix narrative, dont certains narratologues récusent la pertinence. Ainsi, par exemple, Monika Fludernik (2001 : 622) dénonce-t-elle le manque de rigueur de la métaphore de la voix chez Gérard Genette (1972, 1983), où elle perçoit l'application indue d'un modèle communicationnel aux textes littéraires écrits. ${ }^{1}$ Quant à l'idée d'une voix de l'auteur impliqué, elle est au cour de polémiques toujours vivaces; même si, dans ce cas, plutôt que l'inconsistance présumée de la métaphore vocale, c'est la question des critères de définition de l'instance qui en serait le support qui se trouve au cœur des débats.

Mais n'anticipons pas. Avant d'évoquer sa contestation, il peut être utile de présenter sommairement la "vulgate " narratologique en la matière, telle que des tentatives de formalisation variées, mais effectivement soustendues par un modèle communicationnel, ont permis de l'esquisser : celles de Wayne C. Booth (1961 et 1983), Mieke Bal (1977), Jaap Lintvelt ([1981] 1989), Dorrit Cohn ([1978] 1981), Gérard Genette (1972, 1983), Philippe Hamon (1996), etc. - dont on peut également rapprocher les travaux de spécialistes de linguistique textuelle comme Dominique Maingueneau (2004) et Alain Rabatel (2009). En dépit des différences indéniables qui ponctuellement les séparent, en particulier sur la question épineuse de l'articulation de la voix et du point de vue, tous ces chercheurs paraissent partager le constat suivant : le récit de fiction serait tissé de voix multiples. En outre, par-delà la question de leur nombre, puisque chacune d'entre elles peut se révéler porteuse de savoirs et de valeurs spécifiques, doit être posée celle de leur hiérarchisation.

De ces hypothèses partagées découle la convocation fréquente d'une autre métaphore, empruntée au domaine musical, celle de la polyphonie du texte littéraire - qu'entend précisément scruter le présent numéro d'Arborescences. Or, pour qui désire formaliser cette complexité "polyphonique» ou "polyvocale» du récit fictionnel, le cas de figure en apparence particulier où l'instance narrative dont nous (lecteurs) sommes tributaires pour accéder à l'univers de l'histoire racontée, apparaît, pour diverses raisons, suspecte, peut constituer un angle d'attaque privilégié. En effet, le défaut de fiabilité du narrateur pose avec une acuité remarquable la question de la hiérarchie des voix comme des valeurs dont elles sont porteuses - suscitant ainsi une interrogation corollaire sur la notion d'autorité textuelle. En outre, à l'époque contemporaine, le récit à narrateur non fiable et/ou indigne de confiance ne constitue plus une rarissime exception que sa

${ }^{1}$ Pour un compte rendu plus détaillé des critiques adressées par Fludernik à Genette, voir Patron : 137 . 
singularité désignerait à la seule curiosité perverse (?) des amateurs de configurations narratives tératologiques. Au contraire, tout au long du $\mathrm{XX}^{\mathrm{e}}$ siècle, comme en ce XXI ${ }^{\text {e }}$ siècle commençant, les exemples s'en multiplient, en particulier dans le champ de la littérature d'expression française; au point qu'il peut paraître légitime d'y voir l'un des vecteurs privilégiés par les littérateurs de la modernité puis de la postmodernité pour problématiser, voire pour redéfinir (fût-ce par l'absurde) les modalités de la " communication " littéraire. À tout le moins, la question mérite d'être posée ; d'autant plus qu'à quelques rares exceptions près, l'immense majorité des travaux portant sur cette problématique ont été le fait de chercheurs anglosaxons. Il n'est donc que temps de réfléchir, en français dans le texte, aux implications de l' « unreliable narrator »...

Cet effort de réflexion apparaît d'autant plus nécessaire que, si le récit littéraire témoigne d'une forme de polyphonie, les échanges sur le défaut de fiabilité de l'instance narrative tournent bien souvent pour leur part à la cacophonie, tant les présupposés sous-tendant les opinions des parties en présence se révèlent, à l'évidence, inconciliables. Pour tenter, sinon d'apaiser ces débats, du moins d'y voir un peu plus clair, on procédera en deux temps. Sous l'intitulé de "Théorie(s) » sera tout d'abord esquissé un état des lieux des positions défendues en la matière par les tenants de l'approche "rhétorique " (Booth 1961 et 1983), de la narratologie structurale (Lintvelt [1981] 1989, Jouve 2001) et de la narratologie cognitiviste (Nünning 1999, 2005). Davantage qu'une tentative d'arbitrage entre les positions souvent divergentes voire franchement antagonistes des uns et des autres, cette première étape métathéorique fournira l'occasion de poser plusieurs questions de fond - au premier rang desquelles celle des critères indispensables à la formulation d'un diagnostic de non fiabilité narratoriale. Quelles que soient les "réponses" obtenues à l'issue de cette première partie de l'enquête, dans un second temps, sous l'intitulé de "Pratique(s) ", seront envisagées diverses typologies existantes (Mercier et Fortier 2011, Langevin 2011), en vue de mieux cerner la diversité des cas de figure empiriques rassemblés sous la catégorie du "non fiable », sur le double plan sémiotique et pragmatique. On espère ainsi qu'à l'issue de ce parcours, les tenants et aboutissants esthétiques autant qu'épistémologiques du récit à narrateur non fiable et/ou indigne de

\footnotetext{
${ }^{2}$ En français, " unreliable " est traduit tantôt par "non fiable ", tantôt par " non crédible ", voire par "indigne de confiance». Dans la mesure du possible, je tenterai ici de respecter la terminologie proposée par A. Nünning (1999), qui réserve l'appellation de "unreliable » (non fiable) à la non fiabilité factuelle du narrateur, et désigne par " untrustworthy " (indigne de confiance) la non fiabilité idéologique de cette instance, c'est-à-dire la défense par le narrateur de valeurs jugées problématiques en raison de leur caractère inusuel.
} 
confiance auront tant soit peu pu être clarifiés - de même, par la bande, que la question de la polyphonie du texte littéraire. ${ }^{3}$

\section{Théorie(s)}

L'origine des réflexions sur le manque de fiabilité de l'instance narrative en régime fictionnel doit être recherchée dans les travaux de Wayne Clayton Booth. La position de l'auteur de Rhétorique de la fiction (1983) et de "Distance et point de vue " ([1961] 1970, 1977) relève notoirement d'une théorie de l'écart, puisque, selon lui, le paramètre définitoire du narrateur non fiable consiste en une distance perceptible, séparant cette instance de l'auteur impliqués, défini comme second moi de l'auteur réel. Telle est en effet la prémisse du raisonnement de Booth :

Même un roman dans lequel aucun narrateur n'est représenté suggère l'image implicite d'un auteur caché dans les coulisses, en qualité de metteur en scène [...]. Cet auteur implicite est toujours différent de "l'homme réel " - quoi que l'on imagine de lui - et il crée, en même temps que son œuvre, une version supérieure de lui-même. ("Distance et point de vue » : 92-93)

La dimension communicationnelle d'un tel modèle est d'emblée évidente, puisque selon Booth, c'est bien avec ce "second moi" de l'auteur que dialogue le lecteur. On observera en outre que, dès l'origine, l'invention de cette entité anthropomorphe repose sur des présupposés psychologiques, l'auteur implicite (ou impliqué) étant également défini comme "une version de l'auteur extrêmement raffinée et purifiée, plus avisée, plus sensible, plus réceptive que la réalité " ("Distance et point de vue»: 93). Enfin, un souci d'ordre éthique préside également à la création de cette instance, que Booth finit par identifier aux normes et valeurs de l'œuvre. En attestent tout particulièrement les phénomènes de discordance axiologique au sein du récit, comme la définition de l'" unreliable narrator » à laquelle leur prise en compte aboutit :

[...] je dirai d'un narrateur qu'il est digne de confiance (reliable) quand

il parle ou agit en accord avec les normes de l'œuvre (ce qui revient à

\footnotetext{
${ }^{3}$ Question qu'abordent par ailleurs plus «frontalement » les autres contributions réunies dans ce dossier.

${ }^{4}$ Je présenterai la position de Booth à partir de cet article, qui a l'avantage d'avoir été traduit en français - même si cette traduction ne me paraît pas toujours des plus heureuses.

${ }^{5}$ Je privilégierai pour ma part cette traduction de la formule "implied author ", que d'aucuns préfèrent traduire, on le verra sans tarder, par «auteur implicite».
} 
dire : avec les normes implicites de l'auteur [ou : avec les normes de l'auteur implicite $\left.{ }^{6}\right]$ ), et je le dirai indigne de confiance (unreliable) dans le cas contraire [...]. ("Distance et point de vue »: 105)

Sans doute une telle définition conjointe de l'auteur impliqué et du narrateur non fiable n'est-elle pas exempte de défauts, et les détracteurs de Booth ont-ils eu la partie belle au moment d'en dénoncer les flottements. Prête ainsi tout d'abord le flanc à la critique une entreprise définitionnelle qui ne distingue pas suffisamment, dans le cas de l'auteur impliqué, ce qui relève d'une activité artistique (la production/projection par l'auteur d'une image de lui-même), de son pendant esthétique (la réactivation de cette image par le lecteur) et des propriétés textuelles (l'axiologie du récit). Mais, après tout, tels sont bien les trois pôles fondamentaux de l'expérience littéraire ${ }^{8}$, dont Booth respecte dès lors, fût-ce au prix d'une certaine "confusion", la complexité constitutive. Plus embarrassante est à mes yeux la propension déjà signalée à l'anthropomorphisation de ces instances textuelles, de même que leur appréhension en termes psychologiques et éthiques - sans même évoquer certaines dérives prescriptives?.

Reste qu'il est aisé, avec le recul du temps, de pointer les carences d'une entreprise pionnière telle que celle de Booth. Aussi, tout en reconnaissant le caractère perfectible de ce modèle théorique, peut-on à l'inverse mettre l'accent sur plusieurs des intuitions stimulantes qu'il recèle et qui contiennent en germe son propre dépassement. Ainsi, tout d'abord, Booth souligne à juste titre les implications pragmatiques de la construction d'un narrateur non fiable, dont l'impact sur le lecteur est en effet considérable, dans la mesure où son rapport à l'œuvre s'en trouve profondément modifié ("Distance et point de vue»: 105). Ensuite, on peut être sensible à la volonté d'éviter la réduction biographique qui préside à l'invention de l'auteur impliqué, présenté comme distinct de l'auteur réel même si l'anthropomorphisation de cette figure n'est pas sans altérer la clarté de la distinction. En outre, Booth souligne avec pertinence l'extrême diversité des manifestations textuelles (du mensonge à la méprise, sur fond d'une grande variété de régimes ("Distance et point de vue»: 106)) du défaut de fiabilité de l'instance narrative, de même que la multiplicité des fonctions et des effets du procédé. L'un des mérites de l'entreprise tient en effet à la volonté d'articuler analyse des mécanismes textuels et prise en compte des opérations de réception; ce qui voue Booth à l'introduction de nuances justifiées, lui permettant généralement d'éviter l'écueil du dogmatisme. Ainsi signale-t-il

${ }^{6}$ Selon la traduction, en effet préférable, que propose Patron : 144 .

7 À la suite de la distinction introduite par Nünning (1999), mieux vaudrait traduire " unreliable " par " non fiable ». Voir ici même la note 5.

${ }^{8}$ Auxquels on ajoutera toutefois le contexte.

${ }^{9}$ En conclusion de l'article (p. 111-112) en particulier. 
par exemple que " parfois il est presque impossible de décider si le narrateur se trompe, et à quel degré il le fait» ("Distance et point de vue» : 107). De même ne peut-on selon moi, sauf cas d'espèce, que ratifier l'idée que les récits à narrateur non fiable " exigent beaucoup plus de perspicacité de la part du lecteur que ne l'exige une narration en laquelle on se fie au narrateur" ("Distance et point de vue»: 107), puisque le soupçon porté sur l'instance qui médiatise notre accès à la fiction constitue en soi une incitation à la vigilance, et un vecteur de distanciation réflexive. Enfin, aussi embarrassante que puisse parfois paraitre la propension de Booth à raisonner en termes psychologiques et/ou éthiques, cette démarche lui permet toutefois d'effleurer une problématique cruciale pour qui s'intéresse à la réception du récit à narrateur non fiable et/ou indigne de confiance : la possibilité que le lecteur accepte d'être trompé sans pour autant que sa sympathie pour l'instance qui le leurre en pâtisse. Bref, non dénuée de défauts, la réflexion de Wayne C. Booth n'en présente pas moins à l'inverse nombre de qualités : que chacun(e) établisse donc la balance des unes et des autres, en fonction de ses propres convictions épistémologiques et méthodologiques.

Pour l'heure, et sans qu'il s'agisse de dresser un panorama exhaustif des débats sur la question ${ }^{10}$, avant d'évoquer les détracteurs de la pensée de Booth, il peut être utile de rappeler comment certains adeptes de la narratologie structurale s'en sont inspirés, tout en tentant d'en atténuer les défauts. Si Gérard Genette (1983 : 93-107) évacue purement et simplement la notion $d$ ' "auteur impliqué », qu'il juge surnuméraire et/car dénuée de nécessité, d'autres narratologues se refusent à cet usage sans concession du rasoir d'Occam. Ainsi de Jaap Lintvelt ([1981] 1989) qui, dans son Essai de typologie narrative, s'il préfere pour sa part la dénomination $\mathrm{d}$ '" auteur abstrait ", n'en rejoint pas moins l'opinion de Wayne C. Booth : pour lui aussi, cette instance surplombante, qui a barre sur le narrateur comme sur les personnages, s'identifie à l'ensemble des choix de composition et d'écriture, et peut donc être déduite de la forme globale du texte. En outre, par-delà ce point de convergence, Lintvelt apporte une précision d'importance, lorsqu'il affirme que, contrairement au narrateur (dont c'est même la caractéristique définitoire), l'auteur abstrait "ne peut pas intervenir de façon directe et explicite dans son œuvre littéraire comme sujet énonciateur» (26). Pour qui s'intéresse à la polyphonie du texte littéraire, cet élément de définition mérite d'être souligné, car il révèle que ce n'est que de façon plus métaphorique encore que de coutume qu'il est possible de parler de la "voix " de l'auteur impliqué ou abstrait. En effet, si le narrateur du récit de fiction dispose encore de l'équivalent littéraire d'une voix, puisque les mots du texte constituent sa

${ }^{10}$ Je dois de nouveau renvoyer ici à la thèse doctorale d'Anaïs Oléron, qui intégrera un état des lieux, beaucoup plus complet que celui-ci, des débats sur la question du narrateur non fiable et/ou indigne de confiance. 
parole, l'instance qui le coiffe ne peut être au mieux créditée que d'une voix " muette » - ce qui a tout de même toutes les apparences d'un paradoxe... Sauf à raisonner comme ce quidam, évoqué par Stanley Fish, et qui, au cours d'une dispute conjugale, objecterait à son épouse: "Ce n'est pas toi, c'est ta mère qui parle» (2007 : 135). Dans le cas du texte littéraire également, la parole du narrateur serait doublée en sous-main, ou "ventriloquée " (si l'on ne craint pas d'empiler métaphore sur métaphore...) par la parole de l'auteur impliqué ou abstrait. Reste que si la voix du narrateur est accessible à tout lecteur linguistiquement compétent, puisqu'il suffit de savoir lire pour la percevoir, tel n'est pas le cas de la "voix" silencieuse de l'auteur impliqué, qui ne s'esquisse en quelque sorte qu'en filigrane du discours qu'elle sous-tend. Plus que les risques d'acouphènes, d'ordinaire limités, c'est la pratique de la dérive métaphorique qui peut ici faire problème, en obscurcissant ce que la théorie devrait bien plutôt clarifier. Mais ne pas s'accorder sur la pertinence d'une analyse de l'auteur impliqué en termes de voix ne suffit pas à présenter cette instance comme une chimère: que, dans un certain nombre de récits au moins, le narrateur primaire soit supplanté par une instance hiérarchiquement supérieure, est au contraire indéniable.

C'est ce qui apparaît à la lecture d'une des plus convaincantes entreprises de formalisation de la question de la voix dans le récit de fiction : "Qui parle dans le récit ? ", par Vincent Jouve (2001), où se donne à lire, entre narratologie et théorie de la lecture, une réhabilitation de l'auteur impliqué. En effet, à l'issue d'une synthèse des arguments des détracteurs et des défenseurs de la notion, Vincent Jouve se rallie très clairement à l'opinion de Booth et Lintvelt, et estime que "sitôt qu'on se place du côté du lecteur, l'auteur impliqué se présente [...] comme une notion incontournable " (85), puisque c'est avec elle que le lecteur communiquerait. Cette conclusion est en partie fondée sur un examen attentif des relations entre narrateur et auteur impliqué, dont la distinction est ici présentée comme nécessaire dans tous les cas de figure, aussi bien en relation de personne hétérodiégétique qu'homodiégétique. Vincent Jouve démontre la nécessité de cette dissociation sur le plan sémantique (l'auteur impliqué en sait plus que le narrateur, se montre moins crédule que lui, s'en démarque généralement en ne croyant pas à la vérité de l'univers fictionnel, ne défend pas nécessairement les mêmes valeurs) comme sur le plan formel (où, contrairement au narrateur, l'auteur impliqué "ne s'énonce jamais directement» (84)). De plus, si cette réflexion se révèle particulièrement précieuse en l'occurrence, c'est parce que l'auteur impliqué y est présenté comme " une instance plus fiable que le narrateur [...] [puisque] c'est lui qui décide du degré de confiance à accorder à l'instance narrative" (83, je souligne), en en suggérant une évaluation sur les plans esthétique, affectif et idéologique (87) - ce que permet de démontrer l'analyse (87-89) des phénomènes de déphasage énonciatif dans un extrait de Belle du Seigneur (1968) d'Albert Cohen, où sont en effet présents plusieurs des "signaux d'alerte» que Philippe Hamon (1996) estime propres à l'ironie. 
Bref, à la question qu'il avait lui-même posée : "avec qui communique le lecteur ?", à l'issue de son enquête, Vincent Jouve répond sans ambiguïté : " avec l'auteur impliqué ", et insiste sur la réconciliation de la poétique et de la théorie de la lecture qu'autorise selon lui cette notion.

À l'examen, "Qui parle dans le récit ?" constitue donc une synthèse particulièrement lucide des réflexions sur l'auteur impliqué, dont les présupposés épistémologiques sont clarifiés. Vincent Jouve reconnaît ainsi que la notion peut passer pour inutile si l'on est prêt à prendre en compte le horstexte, puisque la voix surplombante pourrait alors être identifiée comme celle de l'auteur réel; mais il la juge a contrario indispensable si l'" on choisit d'analyser les effets de lecture en restant dans un cadre strictement textuel " (85). Tel est, on l'aura compris, son parti pris méthodologique, fondé sur le souci d'étudier la façon dont le lecteur construit sa réception, à partir de la position que lui assignent l'ensemble des «stratégies narratives objectivement mises en œuvre" (76) dans/par le texte. Une telle démarche paraît dès lors guidée par le souci d'endiguer les dérives relativistes, auxquelles sont tout particulièrement exposés les travaux sur la lecture littéraire, par une prise en compte scrupuleuse de la lettre du texte - conformément à la réconciliation ou synthèse méthodologique que Vincent Jouve appelle de ses vœux.

On l'aura constaté, si la position défendue dans "Qui parle dans le récit?" rejoint assez largement celle de "Distance et point de vue ", en particulier lorsqu'il y est affirmé que " quand un narrateur apparaît comme douteux, c'est toujours par rapport aux normes d'un auteur impliqué, c'est-àdire aux normes de l'œuvre" (Jouve : 83), l'entreprise de Vincent Jouve peut être exonérée des défauts qui grevaient celle de Wayne C. Booth. Est-ce à dire que ce louable effort de formalisation théorique ne peut qu'entraîner l'adhésion ? Oui... mais à la seule condition de partager ses postulats de départ - qui, on s'en serait douté, risquent fort de ne pas faire l'unanimité. Par exemple, il est fort peu probable que les opposants à la théorie communicationnelle du récit valident une affirmation telle que "C'est un fait que tout lecteur essaie de reconstruire une figure de l'auteur, ne serait-ce qu'en raison de la structure de communication dans laquelle tout je suppose un $t u$ " (Jouve : 81 , je souligne). Ou encore, la définition de l'auteur impliqué comme un strict effet de texte (Jouve : 79) a toutes les chances de diviser les théoriciens.

De fait, un an avant que Vincent Jouve ne présente la communication ${ }^{11}$ qui deviendra "Qui parle dans le récit ?", Ansgar Nünning avait publié un article destiné à faire date, où il récuse pour sa part non sans virulence les thèses de Wayne C. Booth : "Unreliable, Compared to What? Towards a Cognitive Theory of Unreliable Narration: Prolegomena and

${ }^{11}$ À l'occasion du colloque consacré à la voix narrative par le Centre de narratologie appliquée de l’Université de Nice Sophia-Antipolis, en 2000. 
Hypothesis ${ }^{12} »$. Selon lui, en effet, la question centrale est celle des critères ("standards") susceptibles d'autoriser le critique à formuler un diagnostic de non fiabilité narratoriale. Or il dénie fermement la capacité de jouer ce rôle de paramètre discriminant à l'auteur impliqué de Booth, qu'il juge mal défini, et va jusqu'à présenter comme un fantôme anthropomorphisé, et un " passepartout» critique. Sans entrer dans le détail de ces critiques pour le moins vigoureuses, disons que Nünning blâme essentiellement la relation tautologique instituée entre narrateur non fiable et auteur impliqué ; l'absence d'attention portée à la différence entre non fiabilité factuelle (déformation des faits narrés) et idéologique (défense de valeurs inusuelles), qu'il propose de suppléer en introduisant un distinguo entre non fiable (" unreliable ") et indigne de confiance (" untrustworthy»); l'occultation par l'emploi de métaphores imprécises (" lire entre les lignes", "communiquer dans le dos du narrateur "...) des modalités d'établissement du défaut de fiabilité narratoriale par le lecteur; l'absence de perception de la subjectivité qui préside à l'identification d'un narrateur non fiable et/ou indigne de confiance, etc.

À l'inverse, il estime que la notion d'ironie dramatique permet de mieux rendre compte du phénomène de la narration non fiable, dont, en se fondant en particulier sur les travaux d'Ann Banfield ([1982] 1995) et de Monika Fludernik (1993), il démontre qu'il est possible de repérer divers indices linguistiques et plus généralement textuels (contradictions internes, divergences de points de vue, marques de subjectivité, etc.). Toutefois, selon Nünning, dont c'est là l'idée maîtresse, la question de la narration non fiable ne saurait trouver de réponse aussi longtemps que l'on se cantonne dans les limites d'une approche purement textualiste, ou si l'on préfère "immanente ", dans la mesure où ce phénomène advient à la croisée des opérations d'écriture et de lecture. C'est précisément au nom de cette conviction qu'il préconise un réexamen de la question à la lumière de la théorie des cadres ("frames"), occasionnant ainsi ce que Sylvie Patron présente comme « le tournant cognitif de la narratologie " (Patron : 135-147).

Selon Nünning, en effet, c'est en se référant à un ensemble de présupposés relevant pour partie de l'expérience mondaine, pour partie de la connaissance de la littérature, que le lecteur peut être conduit à considérer un narrateur comme non fiable et/ou indigne de confiance. Du premier type de cadres relèveraient par exemple les savoirs relatifs au monde et aux "normes " sociales, morales, comportementales, psychologiques, linguistiques qui ont cours à une période historique donnée, à quoi viendrait s'ajouter l'axiologie idiosyncrasique de chacun(e); du second les genres, codes, conventions, stéréotypes littéraires, de même que les normes et valeurs mises en place

12 Pour une synthèse critique plus détaillée de la position adoptée par Nünning dans cet article, voir les observations de Patron: 143-147, que je me contente pour l'essentiel de condenser. 
dans/par le texte concerné. Mais Nünning est très loin de se contenter de remplacer l'auteur impliqué par ces cadres ou scénarios de deux ordres. En fait, à la faveur de cette substitution, c'est le poids relatif de l'auteur, du texte et du lecteur qui se trouve profondément modifié. Là où Wayne C. Booth, et après lui les tenants de la narratologie structurale, raisonnaient en termes de repérage d'un procédé textuel perçu comme reliquat d'une activité d'écriture antérieure, Ansgar Nünning considère pour sa part bien plutôt la postulation d'un narrateur non fiable comme un processus interprétatif du lecteur et/ou du critique - selon un réajustement épistémologique qui n'est pas sans évoquer les partis pris théoriques de Stanley Fish ${ }^{13}$.

Cet aggiornamento repose principalement sur la notion de " naturalisation », empruntée à Jonathan Culler (1975 : 134 et passim) :

The construction of an unreliable narrator can be seen as an interpretative strategy by which the reader naturalizes textual inconsistencies that might otherwise remain unassimilable. (Nünning 1999: 69)

La construction d'un narrateur non fiable peut être perçue comme une stratégie interprétative par laquelle le lecteur naturalise les incohérences textuelles qui sans cela demeureraient inassimilables. (Je traduis)

Autrement dit, confrontés à ce qu'ils perçoivent comme autant de contradictions internes insolubles, les lecteurs et/ou critiques les attribueraient à un narrateur non fiable, qu'ils contribueraient par là même à édifier. Aussi, plutôt que d'un procédé d'écriture, s'agirait-il d'une opération de lecture, assimilable à un "mécanisme d'intégration" (Yacobi 1981: 119). Le narrateur non fiable serait alors la résultante des efforts interprétatifs que déploie le lecteur pour résorber ou neutraliser ("naturaliser») ce qui lui apparaît comme autant de dissonances incompréhensibles, sauf à recourir à une telle hypothèse. Or, on le comprend, en la matière, il ne saurait être question d'objectivité, puisque l'idée d'une dissonance repose sur un écart avec des normes et des valeurs par essence subjectives, quand bien même elles seraient pensées comme universelles par celui/celle qui y adhère. Aussi Nünning a-t-il beau jeu de montrer que, sur le plan axiologique en particulier, le "repérage" par maints critiques d'un narrateur non fiable ${ }^{14}$ pour cause de transgression du "sens commun", des "critères moraux normaux" ou de la "décence humaine" relève en fait d'une activité

\footnotetext{
${ }^{13}$ Comme le souligne A. Oléron.

${ }^{14}$ En fait indigne de confiance, d'après la distinction opérée par Nünning lui-même, puisque il est ici question de valeurs. Sa démonstration me paraît d'ailleurs beaucoup plus aisée à produire à propos du narrateur indigne de confiance qu'à propos du narrateur non fiable. Plus généralement, sa réflexion n'est pas exempte de flottements embarrassants, en raison de l'assimilation indue de ces phénomènes, qui en toute rigueur devraient être distingués.
} 
interprétative fondée sur des valeurs contingentes indûment hypostasiées en normes absolues. Plutôt que par rapport à l'auteur impliqué, le narrateur est alors décrété non fiable par rapport à la vision que se fait le critique de la " normalité morale " ou du "sens commun ". D'où, en conclusion, et sur un plan plus général, cette redéfinition du narrateur non fiable, sous forme de réponse à la question posée dans le titre de l'article, "Unreliable, Compared to What?" :

Unreliable, not compared to the implied author's norms and values, but to the reader's or critic's preexisting conceptual knowledge of the world. (Nünning 1999 : 70)

Non fiable, non pas par comparaison avec les normes et les valeurs de l'auteur impliqué, mais avec la connaissance conceptuelle préexistante du monde que possède le lecteur ou le critique. (Je traduis)

Reste enfin à préciser que, loin d'être vécu comme une calamité, le renoncement à l'objectivité tourne chez Nünning à la profession de foi :

Many historians have at last begun to reject the noble dream of objectivity, and it seems to be high time that the narratologists did the same. (Nünning 1999 : 70)

De nombreux historiens ont finalement commencé à renoncer au noble rêve de l'objectivité, et il semble qu'il soit grand temps que les narratologues fassent de même. (Je traduis)

On constate donc que, sur la question de l'auteur impliqué, comme sur celle du rapport du critique à l'objectivité, les positions respectives d'Ansgar Nünning et de Vincent Jouve sont diamétralement opposées, et pour tout dire incompatibles; ce qui, de prime abord, pourrait paraître curieux, puisque tous deux s'efforcent de (ré-)concilier narratologie et prise en compte du lecteur. Mais, à y regarder de plus près, le travail de l'un (Jouve) s'inscrit dans les théories de l'effet, attentives à la pré-orientation de l'activité lectrice par les structures textuelles ${ }^{15}$; au lieu que la réflexion de l'autre (Nünning) relève plutôt des théories de la réception, assez largement émancipées d'un tel conditionnement structurel ${ }^{16}$. Sans doute est-ce ce qui permet de comprendre qu'à partir d'un même souci méthodologique, les deux chercheurs élaborent des modèles théoriques en définitive antagonistes.

Mais qu'en est-il de la fidélité de chacun d'eux à ses présupposés de départ ? Chez Jouve, force est de constater la cohérence du raisonnement à l'égard de ses prémisses: la réhabilitation de l'auteur impliqué découle alors

${ }^{15}$ Dans la lignée des théories de W. Iser [1972] 1985.

${ }^{16}$ Distinction effectuée par A. Oléron dans sa thèse doctorale. 
$\mathrm{du}$ souci d'analyser l'inscription de la lecture, en évitant toute dérive subjectiviste et relativiste. Aussi n'a-t-il aucune raison d'envisager telle lecture idiosyncrasique, qui verrait par exemple un lecteur pédophile récuser le défaut de fiabilité du narrateur du Lolita ([1955] 1959) de Nabokov. En revanche, on peut se demander si l'entreprise de Nünning est au même degré conséquente. En effet, comment concilier l'idée que le diagnostic de non fiabilité narratoriale dépend des normes et valeurs individuelles du lecteur/critique et l'insistance sur l'existence d'indices textuels de discordance ou de dissonance? Se manifeste ainsi une forme de flottement épistémologique; sauf à considérer que c'est précisément le lecteur ou le critique qui construit lesdits indices - ce que, sauf erreur ou omission de ma part, Nünning ne fait pas ${ }^{17}$. Cette façon de ménager les positions respectivement textualiste et cognitiviste peut donc paraître, du moins pour partie, sujette à caution. Et je ne suis pas persuadé que les tentatives plus récentes de Nünning pour amender ses positions initiales, à la lumière notamment des observations de James Phelan (2005), suffisent à dissiper ce flottement. Reconnaître, comme il le fait dans "Reconceptualizing Unreliable Narration : Synthesizing Cognitive and Rhetorical Approaches ", "l'existence d'un agent créateur qui nourrit le texte et le discours du narrateur d'un vaste ensemble de signaux explicites et d'invitations suggestives en vue d'attirer l'attention des lecteurs sur la confession involontaire que livre le narrateur et sur son absence de fiabilité18 $"(2005$ : 104) paraît même accuser l'écart avec l'hypothèse d'une définition du narrateur non fiable et/ou indigne de confiance comme "processus interprétatif». Aussi, plutôt qu'en une authentique synthèse méthodologique, la démarche de Nünning peut-elle paraître consister en la juxtaposition de deux démarches distinctes :

$\left.1^{\circ}\right)$ repérer aussi précisément et rigoureusement que possible les divers indices textuels de non fiabilité ;

$2^{\circ}$ ) étudier la façon dont les lecteurs/critiques peuvent y réagir en vertu de leurs systèmes de normes et de valeurs.

Mais, en dépit de son caractère composite, peut-être cette façon de procéder constitue-t-elle la seule solution pour réconcilier, et articuler, théories de l'effet (d'obédience narratologique) et théories de la réception (d'obédience cognitiviste $\left.{ }^{19}\right)$ ?

\footnotetext{
${ }^{17}$ À la différence de Fish 1980.

${ }^{18}$ Texte original ("[...] the existence of a creative agent who furnishes the text and the narrator with a wide range of explicit signals and inference invitations in order to draw reader's attention to a narrator's unwitting self-exposure and unreliability") traduit par A. Oléron.

${ }^{19}$ D'ailleurs, le présent article pourrait sans doute encourir un reproche similaire, puisque, de la première à la seconde partie, on y observera une forme de glissement apparent des théories de l'effet aux théories de la réception. Cette inflexion méthodologique paraît inévitable, car s'il n'est de théorie que du général, il n'est de critique que du particulier. Sur cette question
} 
Quoi qu'il en soit, ce parcours cavalier vient largement cautionner le verdict d'Antoine Compagnon, selon qui, en matière de théorie de la littérature, "plusieurs réponses sont possibles, non compossibles, acceptables, non compatibles" (Compagnon : 25). Aussi ne saurait-il être question de concilier les inconciliables, ni a fortiori de réconcilier les irréconciliables. Mais, que l'on soit ou non partisan de sa définition par comparaison avec l'auteur impliqué, le narrateur non fiable pose indéniablement un certain nombre de problèmes, théoriques aussi bien que critiques, qu'il peut être utile d'envisager à présent à titre de prolongement de cet état des lieux (partiel) des réflexions sur la question - engageant, de façon corollaire, celle de la polyphonie du texte littéraire.

Tout d'abord, comme l'affirme Vincent Jouve, il importe de préciser que le narrateur non fiable peut se rencontrer en texte(s) quelle que soit la relation de personne. À lire les travaux spécialisés, on s'avise certes d'une considérable prédominance statistique des exemples prélevés dans les récits homodiégétiques; ce qui n'est guère surprenant, puisque, dans cette configuration, l'instance narrative peut être caractérisée par sa subjectivité, par son point de vue limité, et par son ambivalence statutaire - puisque elle est également personnage de la fiction. Sur une telle base, on assistera donc sans surprise à la possible multiplication de contradictions : entre ses valeurs et celles des autres personnages, entre l'étendue de son savoir et/ou son interprétation de l'histoire et celles d'autrui, entre son discours et ses propres actes $^{20}$. Mais, pour y être moins fréquent, le phénomène peut également survenir en relation hétérodiégétique. Par exemple à la faveur d'interventions ou d'intrusions ponctuelles du narrateur, dont la teneur même laisserait le lecteur sceptique - ce qui correspond selon moi à une altération de la pureté typologique, et à un cas de figure intermédiaire ${ }^{21}$. Toutefois, même en (plus) strict régime d'hétérodiégéticité, la fiabilité de l'instance narrative peut parfois paraître sujette à caution, comme dans Le voyeur (1955) d'Alain RobbeGrillet, où le narrateur "effacé " s'ingénie à gommer une lacune révélatrice dans l'emploi du temps de Matthias, le personnage sur lequel le récit est majoritairement focalisé. Bref, plus fréquent en relation homodiégétique, le

épineuse, et par là même impossible à traiter en détail ici, que l'on me permette de renvoyer à Wagner : 2003.

${ }^{20}$ De ce dernier cas de figure témoigne spectaculairement le narrateur homodiégétique de La télévision (Toussaint : 1997) qui, s’il déploie, sur le plan discursif, un réquisitoire impitoyable contre ce médium, profite, sur le plan actantiel, de la moindre occasion pour regarder la télévision. De ce déphasage résultent la plupart des effets humoristiques du texte.

${ }^{21}$ Certes, l'emploi ponctuel du «je » ne suffit pas à faire du narrateur primaire un personnage à part entière de la fiction ; mais il y a tout de même là introduction d'une subjectivité qui rompt avec l'impersonnalité prédominante de la narration hétérodiégétique canonique. À titre personnel, j'y vois donc une forme d'homodiégétisation de l'hétérodiégétique - si j'ose dire... 
procédé du narrateur non fiable est également possible, et parfois actualisé, en relation hétérodiégétique.

D'ordinaire, la narratologie ne s'embarrasse guère de considérations d'ordre historique. Pourtant, la question de l'historicité du narrateur non fiable ${ }^{22}$, c'est-à-dire également celle des normes sous-tendant son repérage, mérite d'être posée. C'est ce dont témoignent les fluctuations de la réception du Vicaire de Wakefield ([1766] 1844), roman d'Oliver Goldsmith. Comme l'a bien montré Vera Nünning ([1998] 2004), alors qu’à l'époque de publication du texte, le narrateur homodiégétique (le vicaire) était presque unanimement considéré comme digne de foi, au cours des dernières décennies se sont multipliées les lectures dénonçant l'hypocrisie de cette figure, ainsi perçue comme suspecte sur le plan éthique. Cet exemple paraît donc invalider les prétentions objectivistes de certains narratologues, puisque la "même " instance narrative peut être perçue comme fiable par les uns (au XVIII et au $\mathrm{XIX}^{\mathrm{e}}$ siècles), non fiable par les autres (au XX et au XXI siècles), mettant ainsi en lumière le poids de notre communauté interprétative d'appartenance, historiquement déterminée, sur la lecture que nous faisons des œuvres. Sans doute d'aucuns liquideraient-ils le débat en ne retenant que la réception première, jugée plus conforme à l'(hypothétique) intention d'auteur, mais ce serait là faire bon marché de la dynamique de dépragmatisation/ repragmatisation qui fonde l'activité de lecture littéraire, et constitue tout bonnement la condition de la survie transhistorique des œuvres. En mettant au jour l'historicité du diagnostic de non fiabilité narrative, conditionné par l'évolution des "normes" psychologiques et éthiques au fil du temps, l'exemple du Vicaire de Wakefield, tel que l'analyse Vera Nünning, paraît donc plaider en faveur d'un relativisme tempéré.

Mais peut-être les divergences interprétatives en la matière ne sontelles pas seulement fonction de l'inscription historique des diverses communautés de lecteurs; et il est fort possible qu'en synchronie aussi, tel narrateur paraisse fiable aux uns, non fiable aux autres. C'est ce que démontre Pierre Bayard dans plusieurs de ses essais paradoxaux. En particulier, et fût-ce par l'absurde, Qui a tué Roger Ackroyd? (1998) se révèle précieux dans le cadre d'une réflexion sur le narrateur non fiable. Pour la vulgate critique, comme pour l'immense majorité des lecteurs, en effet, le narrateur homodiégétique du roman d'Agatha Christie ([1926] 1927), le docteur Sheppard, constitue le parangon du narrateur non fiable, puisque sa mainmise sur l'activité narrative lui permettrait de dissimuler sa culpabilité dans l'affaire du meurtre de Roger

\footnotetext{
${ }^{22}$ Sur ce point, voir également Cavillac 1995, qui démontre de façon particulièrement convaincante, à partir de l'exemple de l'omniscience narratoriale, que les conditions pragmatiques de la vraisemblance évoluent au fil du temps. En effet, si l'omniscience du narrateur était inacceptable avant le roman réaliste du XIX siècle, depuis lors, banalisée par l'usage, elle a tout bonnement cessé d'être perçue comme problématique par les lecteurs. Cette évolution est rappelée dans Mercier et Fortier : 342 et suivantes.
} 
Ackroyd. Or Bayard démontre, texte à l'appui, la bonne foi de Sheppard et/donc son innocence, avant de désigner un autre coupable. Sans doute y a-til là "fiction théorique", plus précisément construction consciente et délibérée d'une lecture "délirante", entreprise à titre expérimental ; mais même s'il ne s'agit pas de son objectif principal, la démonstration de Bayard a pour conséquence de relativiser l'objectivité présumée du diagnostic de non fiabilité narratoriale. Si la lettre du texte est la même pour tous, les idiosyncrasies présidant aux opérations de sélection et de combinaison des éléments textuels par le sujet-lecteur aboutissent somme toute à autant de «versions » textuelles distinctes. C'est donc cette fois la notion de « paradigme intérieur ${ }^{23}$ " qui jouerait le rôle d'insurmontable obstacle en matière d'identification " objective " d'un narrateur non fiable. L'ironie qui travaille l'énonciation des essais de Pierre Bayard doit certes inciter à la prudence au moment de confronter ses positions paradoxales à celles des tenants de la narratologie structurale ou du cognitivisme, mais Qui a tué Roger Ackroyd? n'en démontre pas moins la possibilité, en synchronie, de divergences interprétatives individuelles, au moment de statuer sur le défaut de fiabilité du narrateur d'un récit littéraire.

Pour autant, quelles que soient les vertus heuristiques de ces réflexions, on voit bien à quelles dérives relativistes pourrait aboutir une telle insistance sur l'irréductibilité des paradigmes intérieurs des différents sujetslecteurs. Si la non fiabilité du narrateur était purement et simplement affaire d'appréciation idiosyncrasique, les débats sur la question perdraient toute raison d'être. Or, s'ils perdurent, c'est parce que les théoriciens intéressés par ce problème s'efforcent, chacun à sa façon, et certes à des degrés divers, de réguler l'activité interprétative du lecteur confronté à la configuration particulière que constitue un narrateur non fiable et/ou indigne de confiance.

C'est qu'en dépit des apparences, la lecture littéraire ne saurait être définie comme une activité totalement solipsiste. En effet, le rapport du lecteur au texte repose sur une présomption d'intentionnalité (Compagnon 1998 : 98), c'est-à-dire sur l'idée, pas nécessairement formulée ni même pleinement consciente, que le texte dans sa configuration actuelle est la résultante d'un "projet" auctorial. Sans qu'il s'agisse de renouer régressivement avec le dogme de l'intention d'auteur, cette hypothèse revient tout simplement à estimer que, lisant un texte littéraire, je pars du principe que l'auteur, en écrivant ce texte, a "voulu faire ${ }^{24}$ " quelque chose ; de sorte que je puis repérer, à même la matérialité de l'écrit, des traces de cette intentionnalité. Ce principe paraît tout particulièrement actif dans la lecture des récits à narrateur non fiable, puisqu'il semble alors logique de rapporter le

\footnotetext{
${ }^{23}$ Bayard $2002: 83$ et passim.

${ }^{24}$ Ce qui ne revient donc nullement à nier la possibilité d'écarts entre le " projet " de départ et sa réalisation.
} 
défaut de fiabilité de cette instance à ce que James Phelan (2005) nomme un " agent créateur ». On a ainsi vu que, se ralliant tardivement - au moins pour partie - à cette opinion, Ansgar Nünning lui-même était alors conduit à considérer les indices de non fiabilité comme autant de signaux explicites et d'invitations suggestives adressés au lecteur. On pourrait d'ailleurs estimer qu'avant même le réajustement de sa position, dès 1999, son insistance sur l'existence de marques textuelles de non fiabilité reposait déjà sur une telle présomption d'intentionnalité - sauf à mobiliser la notion quelque peu byzantine d'intentio operis, ce qui serait revenu pour lui, par un autre biais que Booth, à se rendre à son tour coupable d'anthropomorphisation... Bref, comme l'affirmait déjà Barthes, il semble bien que, dans le cadre de l'activité de lecture, j'aie besoin de l'auteur, fût-ce à l'état de "figure ", "sauf à babiller»(Barthes : 46).

\section{Pratique(s)}

Que les théoriciens souscrivent plutôt à une définition du narrateur non fiable par comparaison avec l'auteur impliqué (Booth 1961 et 1983, Lintvelt [1981] 1989, Jouve 2001), avec les "scénarios ", normes et valeurs du lecteur (Nünning 1999), ou qu'ils adoptent une position intermédiaire ou "synthétique» (Phelan, Nünning 2005), tous paraissent en définitive s'accorder sur un point : la possibilité de repérer en texte divers indices de non fiabilité narratoriale. Aussi, même si les présupposés épistémologiques de la notion demeurent sujets à controverses, peut-on constater au cours des deux dernières décennies la multiplication de typologies poétologiques ambitionnant de distinguer les variantes du phénomène, en vue également d'en interroger la ou les fonctions. Citons par exemple les travaux d'Andrée Mercier et Frances Fortier, qui, dans le cadre de réflexions sur la transmission narrative, sont conduites à distinguer « la narration impossible [qui] renvoie à la compétence [problématique] du narrateur ", "la narration indécidable [qui] renvoie, quant à elle, à l'identité du narrateur ", et "la narration ambiguë, enfin, [qui] renvoie à la crédibilité du narrateur et à la fiabilité de son récit " (Mercier et Fortier: 334-335). Si seul le troisième pôle de cette typologie paraît de prime abord correspondre au sens strict à la question du narrateur non fiable, à l'examen le premier en relève également, puisque un narrateur doté d'une compétence problématique risque fort, par là même, de paraître suspect aux yeux du lecteur - sans même parler des possibles cas de "syncrèse" entre ces divers types. L'intérêt d'une telle entreprise de formalisation est triple: tout d'abord, la densité même du corpus analysé témoigne avec clarté de la fréquence du traitement déceptif de la voix narrative dans le roman de l'extrême contemporain ; ensuite, ces affinements typologiques permettent d'établir que « les conditions de possibilité, de sens et de vérité du récit et de la fiction sont mises en jeu de manière distincte selon 
que l'on fragilise le récit par une "fausse " incompétence du narrateur, que l'on brouille l'origine de la narration ou que l'on mette en doute la valeur du récit par le caractère retors et manipulateur du narrateur" (Mercier et Fortier : 351) ; enfin, par-delà ces distinctions, peut être mis au jour un dénominateur commun d'ordre fonctionnel, puisque dans tous les cas, "la narration, en incarnant, dans sa forme énonciative, son impossibilité, son origine ou sa valeur, n'a de cesse de relancer la dimension transactionnelle du récit " (Mercier et Fortier : 352) - proposant ainsi une redéfinition en acte des modalités de la transmission narrative, plus précisément du pacte romanesque.

Dans une perspective méthodologique proche, mais davantage ouverte sur les normes et valeurs du lecteur, et portant sur le seul récit homodiégétique, Francis Langevin (2011) propose pour sa part d'évaluer le défaut de fiabilité du narrateur à l'aune de la notion d' "exotisme», dans la mesure où un narrateur-personnage peut manifester une forme d'altérité sur le plan culturel et/ou éthique et/ou cognitif. En outre, s'inspirant des travaux de Greta Olson (2003), il distingue deux grandes catégories de narrateurs non fiables, qu'il place sous les auspices des figures emblématiques de Lazarillo et Épiménide. Pour schématiser, les narrateurs du premier type pourraient être caractérisés par leur faillibilité, les narrateurs du second type par leurs dispositions manipulatrices; et susciteraient chez le lecteur une relation plus (dans le premier cas) ou moins (dans le second cas) empathique. À l'examen, ces distinctions paraissent tout à fait pertinentes, tant sur le plan sémiotique que sur le plan pragmatique. Selon moi, on pourrait d'ailleurs aboutir à un diagnostic similaire en recourant à la notion d'ironie. En effet, les narrateurs homodiégétiques que Francis Langevin rassemble sous le patronage de la figure du picaro paraissent tous peu ou prou ironisés; ce qui implique qu'une instance hiérarchique supérieure, en charge de leur conception, établisse ainsi à leur détriment une relation de connivence avec le lecteur - laquelle repose précisément sur la dimension perceptible des carences que manifeste le discours de l'instance narrative. Différemment, les narrateurs homodiégétiques fédérés sous les auspices d'Épiménide le Crétois semblent pour leur part bien plutôt incarner autant de figures d'ironistes, dont la duplicité du discours, potentiellement fourvoyante pour le lecteur, peut par là même se révéler inquiétante. Dès lors, en termes d'effet ou d'impact, peut-on aboutir sensiblement aux mêmes conclusions que Francis Langevin: la faillibilité de nombre de narrateurs-personnages ironisés, loin de leur aliéner les suffrages des lecteurs, est souvent susceptible de générer une relation empathique, à proportion même des insuffisances ainsi obliquement signalées. Pour prendre un cas d'école, chez Conan Doyle ${ }^{25}$, les limitations cognitives de

${ }^{25}$ Dans la série des aventures de Sherlock Holmes (romans et nouvelles), dont le Dr Watson assure le plus souvent la narration, en qualité de «biographe » du Grand Détective. 
Watson, telles que sa narration les révèle, en font un parangon de l'humanité moyenne, en tant que tel éminemment rassurant pour un lecteur qui, pas plus que le bon docteur, ne parvient à comprendre le sens des observations sibyllines que Holmes multiplie au cours de ses enquêtes. Si l'on me passe un vocabulaire aussi psychologisant, à Holmes l'admiration du lecteur, à Watson sa sympathie. À l'inverse, les narrateurs-personnages volontiers portés à l'ironie, comme souvent chez Nabokov et Robbe-Grillet, ne disposent pas a priori du même "capital de sympathie", dans la mesure où le lecteur peut cette fois redouter de faire les frais de leur propension à la manipulation, se traduisant par la tenue d'un discours à double entente.

$\mathrm{Du}$ moins un tel partage typologique est-il globalement recevable, mais ne saurait à lui seul tenir lieu de "grille de lecture ", tant en la matière les cas d'espèce sont légion. Ainsi Bertram Wooster chez Wodehouse ${ }^{26}$ et Charles Kinbote chez Nabokov ([1962] 1965) constituent-ils deux narrateurs homodiégétiques supérieurement ironisés; mais l'incompétence culturelle, lexicale, narrative et actantielle du premier en fait paradoxalement une figure attachante, alors que les œillères du second, aggravées par sa mégalomanie, tendent à lui aliéner la sympathie du lecteur. Pour marquer un point d'accord avec Wayne C. Booth et la plupart des narratologues, il y a bien dans les deux cas ironisation du narrateur par une instance surplombante en charge de sa conception et de l'élaboration de son discours (l'auteur impliqué); pour marquer un point d'accord avec Ansgar Nünning et les cognitivistes, ce dénominateur commun d'ordre structurel ne suffit pas à rendre compte d'un phénomène dont la saisie exige la prise en compte de schèmes expérientiels et de valeurs qui renvoient en définitive à l'appréciation des sujets empiriques que sont les lecteurs. Après tout, rien ne peut m'assurer que mon goût pour Bertram Wooster et mon dégoût pour Charles Kinbote seront unanimement partagés; sauf à supposer que cette appréciation contrastée repose sur des constantes axiologiques transindividuelles et transhistoriques - ce qui est peut-être après tout possible, mais paraît pour le moins délicat à démontrer.

Quoi qu'il en soit de ces possibles fluctuations de la réception, elles ne suffisent pas à récuser le rôle de paramètre discriminant que peut jouer l'ironie sur le plan structurel. Toutefois, valide en relation homodiégétique, la distinction entre narrateur ironisé et narrateur ironisant le demeure-t-elle en relation hétérodiégétique ? De nouveau, il paraît malaisé de l'établir, tant dans cette autre relation de personne, les phénomènes de dissonance ou de discordance qui tiennent lieu d'indices de non fiabilité semblent difficiles à produire. En fait, le problème vient de ce que, en régime d'hétérodiégéticité strict, c'est-à-dire hors intrusions d'un narrateur usant ponctuellement de la première personne, l'instance narrative primaire tend selon moi à se confondre tendanciellement avec l'auteur impliqué. Ainsi, on voit mal ce que

${ }^{26}$ Dans la série des Jeeves, dont Bertram Wooster est généralement le narrateur. 
pourrait être un narrateur hétérodiégétique ironisé, tout simplement parce que, dans cette autre configuration, les défauts de la narration seraient très probablement versés par le lecteur non pas au (seul) passif de l'instance narrative, mais de l'instance en charge de la construction du récit dans son ensemble, narrateur compris - c'est-à-dire au passif du scripteur, de l'auteur impliqué, voire de l'auteur tout court $^{27}$. Peut-être ce type est-il malgré tout réalisable ${ }^{28}$, mais je dois confesser n'en point connaître d'exemple réellement probant; ce qui, au risque de la présomption, ne me paraît pas plaider en faveur de sa dimension usuelle ${ }^{29}$. Dès lors, même si le narrateur hétérodiégétique manipulateur existe indéniablement (en attestent non seulement Le voyeur (1955), mais aussi Un cabinet d'amateur (1979) de Georges Perec et Un an (1997) de Jean Echenoz), ferait défaut l'un des pôles

\footnotetext{
27 Sauf, bien sûr, s'il y a enchâssement narratif, dans le cas d'un narrateur intradiégétique.

${ }^{28}$ Il l'est incontestablement en droit, puisqu'il "suffirait " pour le produire de désolidariser les énoncés mimétiques des énoncés non mimétiques, par exemple les maximes à caractère gnomique et autres jugements de valeur assumés par le narrateur, de sorte que ces derniers paraîtraient sinon irrecevables, du moins suspects, de même, par contrecoup, que l'instance qui en est responsable. De plus, certains théoriciens en repèrent des exemples en texte, comme Cohn ([1999] 2001 : ch. VII et VIII), estimant que le narrateur hétérodiégétique primaire de Der Tod in Venedig (1912) [La Mort à Venise] de Thomas Mann serait indigne de confiance au prix d'une virtuosité interprétative qui me paraît toutefois excéder les compétences herméneutiques de la plupart des lecteurs. Si l'on généralise ce type de démarche, la ténuité des "indices " qu'il s'agirait de repérer et d'interpréter ne risquerait-elle pas alors de nous conduire à voir des narrateurs "non fiables" partout - ou presque ? Dorrit Cohn en est d'ailleurs pleinement consciente (et le confesse (1999: 199)), qui précise ainsi sa position : "Je pense qu'il faut accepter qu'un critique confronté à ce type d'incongruité [désaccord entre langage mimétique, qui raconte l'histoire, et commentaire évaluatif du narrateur] soit toujours libre de l'attribuer à l'auteur plutôt qu'au narrateur. Mais je maintiens que l'intégrité esthétique et idéologique de l'œuvre en question ne peut être sauvegardée que si on opte pour la séparation entre son narrateur et son auteur" (1999: 199). Soit; mais c'est bien ce parti pris qui à mes yeux fait problème, car l'hypothèse d'une « œuvre pleinement achevée, et, en ce sens-là, sans défaut " (Cohn 1999 : 222) me semble discutable, dans la mesure où elle excède largement la simple présomption d'intentionnalité qui fonde l'activité de lecture littéraire. Autrement dit, l'idée de la "perfection" de l'œuvre relève selon moi d'une décision interprétative toute subjective (la mienne, inverse, l'étant bien sûr tout autant), que je juge exagérément optimiste, et par là même bien peu " réaliste ".

${ }^{29}$ En effet, même si l'on souscrit à l'interprétation remarquablement fine et attentive à la lettre du texte que propose Dorrit Cohn de La Mort à Venise, et éprouve sur cette base la tentation de valider l'hypothèse d'un narrateur primaire hétérodiégétique non fiable, sans le secours d'autres exemples similaires, il paraîtrait quelque peu exagéré d'y identifier un type narratif. Cela dit, puisque cette configuration narrative est bel et bien réalisable, rien ne permet d'affirmer que de plus nombreuses occurrences n'en apparaîtront pas à l'avenir. Bref, mon propos n'est pas de désavouer les analyses de Dorrit Cohn, encore moins de récuser la dissociation de l'auteur et du narrateur comme critère de démarcation entre fiction et non fiction qu'elle défend (puisque tel est le cadre dans lequel surviennent ses observations), simplement de faire montre de prudence au moment de considérer l'ironie comme paramètre décisif pour l'établissement du défaut de fiabilité de l'instance narrative d'un texte fictionnel - ce qui n'est pas la préoccupation première de l'auteur du Propre de la fiction.
}

\section{Arborescences \\ Revue d'études françaises}

ISSN: 1925-5357 
permettant d'étendre le critère démarcatif de l'ironie à cette autre relation de personne. Pour reprendre le distinguo proposé par Francis Langevin, en régime d'hétérodiégéticité, si l'on rencontre nombre d' "Épiménide", les "Lazarillo" se font pour le moins beaucoup plus rares. En témoigne d'ailleurs, on l'a vu, la nette prédominance des récits homodiégétiques dans les travaux consacrés au narrateur non fiable. Ces quelques observations incitent donc à nuancer la pertinence de l'auteur impliqué (conçu comme système de normes et de valeurs de l'œuvre, élaboré par l'agent créateur) comme critère de détermination du défaut de fiabilité de l'instance narrative. Parfaitement convaincant dans le cas d'un narrateur faillible, le recours à cette notion l'est en effet beaucoup moins dans le cas d'un narrateur manipulateur qui, on l'a vu, parait alors se confondre tendanciellement avec l'auteur impliqué $e^{30}$.

En fait, les problèmes soulevés par l'établissement des normes d'identification du narrateur non fiable sont tels qu'on peut tout de même se demander s'il ne s'agirait pas là en définitive d'une forme d' «impasse " pour la théorie du récit. D’autant que, depuis au bas mot la deuxième moitié du $\mathrm{XX}^{\mathrm{e}}$ siècle, se sont multipliés les récits reposant sur des codes esthétiques, axiologiques, voire épistémologiques profondément anomiques, de sorte que l'analyse du narrateur selon les standards littéraires et éthiques usuels y devient sinon impossible, du moins fortement problématique. En attestent par exemple le poids de la logique illogique du fantasme sur le traitement de la voix narrative dans Le ravissement de Lol V. Stein (1964) de Marguerite Duras; ou la déceptivité généralisée affectant l'origine énonciative dans le " post-exotisme " d'Antoine Volodine ${ }^{31}$, "xénolittérature " édifiant son propre système de valeurs paradoxal, etc. Ces exemples, parmi bien d'autres, tant les phénomènes similaires se sont multipliés dans la littérature de l'extrême contemporain, incitent donc à poser la question de la mutabilité des normes sur lesquelles nous prenons appui au moment de porter un diagnostic de non fiabilité narratoriale. À tout le moins, cette multiplication paraît constituer une incitation à privilégier les études empiriques, en prêtant une attention particulière à la configuration spécifique de chaque œuvre, comme le recommandait déjà Wayne C. Booth.

Dans cette concentration délibérée de l'attention sur la lettre des textes, d'aucuns blâmeront peut-être une attitude démissionnaire sur le plan théorique ? Une telle crainte ne serait qu'en partie fondée, car par-delà la diversité des configurations narratives, divers points communs d'ordre fonctionnel et pragmatique ont tôt fait d'apparaitre. Tout d'abord, la construction d'un narrateur non fiable peut relever d'une technique originale de captatio benevolentiae, dans le cas du moins d'une faillibilité ostentatoire de

${ }^{30} \mathrm{Du}$ moins en relation hétérodiégétique.

${ }^{31}$ Voir Wagner 2014. 
l'instance en cause. Ainsi nombre de narrateurs homodiégétiques faillibles (héritiers en cela de la tradition picaresque) sont-ils vecteurs d'effets humoristiques, comiques, voire franchement bouffons - tel (de nouveau) Bertram Wooster, dont la réjouissante incompétence généralisée saurait difficilement être égalée. En outre, par-delà ses vertus divertissantes, qu'on aurait tort d'occulter, la figure du narrateur borné participe souvent d'une valorisation plus «subtile » du lecteur. Pour citer d'autres exemples déjà rencontrés, tel est le cas de l'incapacité interprétative de Watson chez Conan Doyle, comme de son "clone" tardif, Hastings, chez Agatha Christie, qui multiplient les aveux d'incompréhension face aux prouesses de leur "maitre " respectif, Holmes pour l'un, Poirot pour l'autre. L'incompétence herméneutique de ces figures vise alors à rassurer le lecteur quant à la sienne propre, qu'il est dès lors fondé à considérer comme excusable, puisque il la partage avec ce témoin privilégié qu'est pourtant le narrateur. Mais il advient ponctuellement que le défaut de sagacité du narrateur homodiégétique relève, comme le signale Nünning, d'une variante de l'ironie dramatique, comme dans tous les cas où le lecteur est censé comprendre ce que l'instance en charge de la narration échoue pour sa part à saisir - ce qui induit un contraste potentiellement gratifiant ${ }^{32}$. Bref, que je me réjouisse du spectacle de son aveuglement complaisamment exhibé, que je me rassure à l'idée que mes perplexités interprétatives sont également les siennes, ou que je savoure la supériorité herméneutique que ses limitations cognitives m'incitent à m'attribuer, le narrateur faillible a toutes les chances de capter ma bienveillance de lecteur : à qui me fait rire et/ou me rassure et/ou me valorise, il sera beaucoup pardonné.

On peut selon moi, au risque du paradoxe, en dire "autant" de certains narrateurs manipulateurs, quelle que soit la relation de personne dont ils relèvent, mais à condition de préciser qu'il s'agit là plutôt de séduction (parfois très indirecte) que de captation de bienveillance au sens strict. En témoigne spectaculairement "Un drame bien parisien" ([1890] 1891) d'Alphonse Allais, nouvelle dont Umberto Eco ([1979] 1985) a démontré à quel point elle programme l'échec de la coopération du lecteur, en l'incitant à sur-interpréter par la convocation "réflexe" du stéréotype intertextuel du flagrant délit de l'amour adultère, avant de se dénouer par une pirouette nonsensique. Lorsqu'il est ainsi planifié par le texte, et soigneusement préparé par les pièges que nous tend un narrateur des plus roués, il est en effet un plaisir paradoxal de l'échec interprétatif, valant incitation à la relecture - ce dont, par-delà cet exemple particulier, conviendront la plupart des lecteurs de récits de détection, incapables d'aboutir à la solution révélée par le détective, mais la savourant d'autant plus. En témoigneraient également des textes très

${ }^{32}$ Par exemple lorsque le lecteur comprend ce que Watson (ou Hastings) ne comprend pas ce qui, espérons-le, se produit tout de même parfois... 
différents sur le plan esthétique de celui d'Allais, comme "Le réquisitionnaire " ([1831] 1832) de Balzac ou "À s'y méprendre " (1893) de Villiers de l'Isle-Adam. Bref, et même si dans les deux cas évoqués le rapport de forces institué entre instance narrative et lecteur apparaît diamétralement opposé, il semble bien que la construction d'un narrateur faillible ou manipulateur procède pour le moins à quelque degré, sinon toujours de la captation de bienveillance du lecteur, du moins de sa séduction, sur le mode direct ou indirect.

Ensuite, et cette fonction n'est nullement incompatible avec la précédente, la construction d'un narrateur non fiable contribue à la "conscientisation", ou si l'on préfère à l'éveil du lecteur. C'est déjà ce que signalait Wayne C. Booth lorsqu'il insistait sur le degré élevé de perspicacité que ce type de configuration narrative exigeait du lecteur, par rapport à la situation où il lui est loisible de se fier au narrateur. En effet, à partir du moment où le texte présente les compétences du narrateur et/ou les valeurs dont il se fait le promoteur comme peu ou prou problématiques, l'attention du lecteur est attirée sur la médiation dont dépend son accès à l'histoire racontée - ainsi dépouillée de son illusoire "évidence ». Autrement dit, il devient alors impossible de croire naïvement que l'histoire se raconterait d'elle-même. En soi, le procédé consistant à inquiéter la fiabilité de l'instance narratoriale constitue donc, en raison de ses vertus dénudantes, un appel à la vigilance, et une vive incitation à adopter une posture de réception distanciée et critique. Sans doute cette capacité distanciatrice est-elle plus évidemment perceptible dans le cas du narrateur manipulateur que dans celui du narrateur faillible, mais elle n'en paraît pas moins inhérente au traitement déceptif de la voix narrative, quelles qu'en soient les modalités. Par exemple, même s'il est fort peu probable que la sensibilisation du lecteur aux propriétés du medium littéraire constitue l'un des objectifs prioritaires de P. G. Wodehouse, il n'empêche que, même dans la série des Jeeves, l'incompétence hypertrophiée du narrateur, Bertram Wooster, génère non seulement des effets comiques, mais aussi, fût-ce de façon secondaire voire involontaire, une mise à nu des procédés de textualisation ${ }^{33}$.

Une fois ce constat formulé, on peut tout de même convenir que certains écrivains ou groupements d'écrivains ont usé fort consciemment et de propos tout à fait délibéré du procédé du narrateur non fiable et/ou indigne de confiance, en vue d'engager une réflexion en acte sur les conditions d'établissement et les modalités de fonctionnement du pacte romanesque, et d'y associer les lecteurs. Dans le champ littéraire français, en témoignent tout particulièrement les évolutions de la pratique narrative depuis 1950 ; à commencer par le "Nouveau Roman ". Dans la mesure où les écrivains de cette mouvance partagent, au moins pour partie, une conception littéraliste de

${ }^{33}$ D'ailleurs ponctuellement renforcée par diverses incidentes métatextuelles. 
l'écriture romanesque, il n'est guère surprenant de constater que, dans leurs œuvres, le traitement anomique de la voix narrative occupe une place de choix. En effet, pour qui souhaite détourner le lecteur des séductions de l'illusion référentielle, et le familiariser à l'inverse avec les procédés de composition et d'écriture, la construction d'un narrateur non fiable et/ou indigne de confiance constitue à l'évidence une technique des plus efficaces au même titre, bien sûr, que l'ensemble des mécanismes dénudants et déréalisants. Dans les œuvres des nouveaux romanciers, le procédé participe en outre d'une plus vaste entreprise de contestation de l'autorité narrative, sur fond de rejet des notions corollaires d'omnipotence et d'omniscience. Que, durant l'ère du soupçon, prolifèrent les narrateurs suspects n'a donc en définitive rien d'étonnant. Ainsi peut-on aisément constater que tous les cas de figure précédemment évoqués se rencontrent dans ces textes: pour ne retenir que quelques exemples parmi les plus connus, si dans L'innommable (1953) de Beckett, l'origine de la voix narrative est brouillée au point d'en devenir indécidable, le narrateur homodiégétique de Molloy (1951) apparaît clairement faillible dès le célèbre incipit du roman; quand (on l'a vu) le narrateur hétérodiégétique du Voyeur (1955) de Robbe-Grillet incarne le type même de l'instance manipulatrice - sans même évoquer La reprise (2001), où la question de la non fiabilité est mise en intrigue, et portée à son comble, via l'affrontement de deux narrateurs rivaux, dont l'un parasite la parole de l'autre à la faveur d'un surprenant dispositif notulaire. Bref, pour notoire qu'elle soit, la fréquence du procédé dans l'esthétique néo-romanesque n'en confirme pas moins, de façon très spectaculaire, sa fonction distanciatrice.

Peut-être aurait-on pu s'attendre à ce que, sur fond de prégnante renarrativisation, les narrateurs non-fiables et/ou indignes de confiance se raréfient dans le roman d' "au-delà du soupçon", disons des années 1980 à aujourd'hui. Or les travaux des spécialistes de l'extrême contemporain permettent de conclure qu'il n'en est rien. Certes, la littérature narrative de ces dernières décennies ne tend plus au même degré à entraver le déroulement de la fabula, ni à obérer le fonctionnement de l'illusion référentielle au nom d'un credo littéraliste non dénué parfois d'une certaine pesanteur didactique ; mais force est de constater que les narrateurs non fiables et/ou indignes de confiance continuent de s'y manifester, voire y prolifèrent de plus belle. De nouveau, tous les types précédemment répertoriés s'y rencontrent : narrateur homodiégétique faillible (Cinéma (1999) de Tanguy Viel, L'ouvre posthume de Thomas Pilaster (1999) d'Éric Chevillard) ou manipulateur (Nous trois ${ }^{34}$ (1992) de Jean Echenoz), narrateur hétérodiégétique manipulateur ( $U n$ an (1997) du même Echenoz), sur fond de résurgence ponctuelle de l'indécidabilité énonciative (le post-exotisme volodinien).

${ }^{34}$ Même si la relation de personne à l'œuvre dans le roman d'Echenoz peut faire débat, qui témoigne à vrai dire d'une forte tension entre homo- et hétérodiégéticité. 
Mais le constat de la permanence du procédé ne doit pas inciter à récuser toute différence entre roman moderne et postmoderne. Il semble en fait que l'inquiétude portée sur la fiabilité du narrateur permette à nombre d'écrivains actuels de produire des fictions tout aussi lucides que celles de leurs devanciers, sans pour autant renouer avec les dogmes et les exclusions en vigueur à l'apogée du modernisme. Y contribue la dimension oblique du procédé, qui loin d'annihiler la fabula, constitue une incitation à en jouir sans fausse honte, mais en toute conscience des propriétés du texte qui la suscite ${ }^{35}$. Telle est également, me semble-t-il, la conclusion d'Andrée Mercier et Frances Fortier (2011), qui identifient dans les narrations indécidables, impossibles ou ambiguës du roman contemporain autant de renégociations en acte du pacte romanesque. Seraient ainsi confirmées non seulement l'existence mais aussi la possible convergence des fonctions que j'ai précédemment attribuées au procédé du narrateur non fiable et/ou indigne de confiance : séduire et « éveiller » le lecteur.

À l'issue de ce long parcours, il est temps de tirer quelques enseignements, qui, plutôt que de la conclusion en bonne et due forme, risquent fort de relever du jugement de Salomon et/ou de la réponse de Normand... En effet, on l'a vu, sur le plan théorique, il serait utopique et pour tout dire vain de prétendre réconcilier les tenants de l'approche rhétorique, de la narratologie structurale et du cognitivisme, dans la mesure où les uns et les autres définissent le narrateur non fiable d'après des paramètres distincts, et en vertu de convictions épistémologiques incompatibles. Pour éviter tout excès normatif en la matière, je proposerais bien de ne plus parler de narrateur non fiable, mais simplement de narrateur suspect; même si j'ai bien conscience qu'il ne s'agit là (du moins pour partie) que d'un artifice rhétorique.

Toutefois, on l'aura également constaté, ces problèmes théoriques insolubles ne constituent pas un obstacle insurmontable à la production d'études empiriques du procédé en cause, dont diverses typologies se proposent de recenser les variantes sémiotiques et pragmatiques, et d'élucider les possibles fonctions. En définitive, peut-être cette apparente contradiction des plans théorique et critique n'est-elle d'ailleurs pas aussi embarrassante qu'on pourrait le croire de prime abord. Il semble en effet possible, sinon de la résoudre, du moins de la dépasser, en optant pour la posture que Raphaël Baroni propose de nommer "critique polyphonique », et qui :

${ }^{35}$ Voir Wagner 2011. 
[...] revient à refuser de trancher le nœud gordien de la polyphonie, refuser également de le démêler, mais tout de même [à] en analyser chaque brin et la manière dont il s'entrelace avec les autres [...] [afin] de mettre en lumière les facteurs (scénographie, intertextualité, posture auctoriale, position des communautés interprétatives) conditionnant la configuration d'un espace interprétatif en tension [...]. (Baroni : 6)

De fait, chacun peut constater que la présence en texte d'un narrateur suspect constitue ipso facto une très vive incitation à réfléchir aux présupposés qui structurent nos interprétations - ce qui dote le procédé d'une potentielle fonction de révélateur épistémologique.

Reste que le narrateur suspect peut non seulement alimenter les controverses théoriques et nourrir les typologies poétologiques, mais aussi dispenser un plaisir spécifique - également digne d'intérêt. Pour le formuler autrement, quand le narrateur boit, boite (dans le cas du narrateur faillible), ou ironise (dans le cas du narrateur manipulateur), le lecteur trinque-t-il? S’il est impossible de répondre catégoriquement à une telle question, compte tenu de la diversité empirique des sujets-lecteurs, du moins peut-on estimer qu'en droit, le narrateur suspect ne constitue pas un obstacle durable au plaisir de lecture - tout au contraire. En effet, faillible, il divertit, rassure et valorise le lecteur; manipulateur, il lui offre la possibilité d'une jouissance plus stratégique. Dès lors, s'il est question de "trinquer", ce devrait être bien plutôt à la santé du créateur de cette instance, dont les inflexions de la voix favorisent une réception à plus d'un titre gratifiante.

\section{Références bibliographiques}

Allais, A. 1891. "Un drame bien parisien ». Dans À se tordre. Paris : Paul Ollendorff : 213-131. [1890].

Bal, M. 1977. Narratologie. Essais sur la signification narrative dans quatre romans modernes. Paris : Klincksieck.

Balzac (de), H. 1832. Le réquisitionnaire. Paris : Gosselin. [1831].

Banfield, A. 1995. Phrases sans parole. Théorie du récit et du style indirect libre. Paris: Seuil. [1982].

Baroni, R. 2014. «La guerre des voix. Critique polyphonique et divergences interprétatives dans l'œuvre de Michel Houellebecq ". Dans COnTEXTES 4. http://contextes.revues.org/5979. Page consultée le 11 décembre 2015.

Barthes, R. 1973. Le plaisir du texte. Paris : Seuil.

Bayard, P. 1998. Qui a tué Roger Ackroyd? Paris : Minuit.

Bayard, P. 2002. Enquête sur Hamlet. Le dialogue de sourds. Paris : Minuit.

Beckett, S. 1951. Molloy. Paris : Minuit.

Beckett, S. 1953. L'innommable. Paris : Minuit.

Booth, W. C. 1970, 1977. "Distance et point de vue ». Dans Poétique du récit, sous la direction de G. Genette et T. Todorov. Paris : Seuil : 85-113 [1961].

Booth, W. C. 1983. The Rhetoric of Fiction. Chicago : University of Chicago Press. [1961]. 
Cavillac, C. 1995. "Vraisemblance pragmatique et autorité fictionnelle ». Dans Poétique 101 : 23-46.

Chénetier, M. 1989. Au-delà du soupçon: la nouvelle fiction américaine de 1960 à nos jours. Paris : Seuil.

Chevillard, É. 1999. L'euvre posthume de Thomas Pilaster. Paris : Minuit.

Christie, A. 1927. Le meurtre de Roger Ackroyd. Paris : Librairie des Champs-Élysées. [1926].

Cohen, A. 1968. Belle du Seigneur. Paris : Gallimard.

Cohn, D. 1981. La transparence intérieure. Modes de représentation de la vie psychique dans le roman. Paris : Seuil. [1978].

Cohn, D. 2001. Le propre de la fiction. Paris : Seuil. [1999].

Compagnon, A. 1998. Le démon de la théorie. Littérature et sens commun. Paris : Seuil.

Culler, J. 1974. "The Elusive Narrator." Dans Flaubert - The Uses of Uncertainty. Ithaca et New York : Cornell University Press : 109-122.

Culler, J. 1975. Structuralist Poetics: Structuralism, Linguistics and the Study of Literature. Ithaca et New York : Cornell University Press.

Duras, M. 1964. Le ravissement de Lol V. Stein. Paris : Gallimard.

Échenoz, J. 1992. Nous trois. Paris : Minuit.

Échenoz, J. 1997. Un an. Paris : Minuit.

Eco, U. 1985. Lector in fabula, ou la coopération interprétative dans les textes narratifs. Paris : Grasset. [1979].

Fish, S. 1980. Is there a Text in This Class? The Authority of Interpretive Communities. Cambridge et Londres : Harvard University Press.

Fish, S. 2007. Quand lire c'est faire. Paris : Les Prairies Ordinaires.

Fludernik, M. 1993. The Fictions of Language and The Languages of Fiction: The Linguistic Representation of Speech and Consciousness. Londres et New York : Routledge.

Fludernik, M. 1996. Towards a "Natural" Narratology. Londres et New York : Routledge.

Fludernik, M. 2000. "Beyond Structuralism in Narratology : Recent Developments and New Horizons in Narrative Theory." Anglistik XI-1 : 83-96.

Fludernik, M. 2001. "New Wine in Old Bottles? Voice, Focalization, and New Writing." New Literary History XXXII-3 : 619-638.

Genette, G. 1972. Figures III. Paris : Seuil.

Genette, G. 1983. Nouveau discours du récit. Paris : Seuil.

Goldsmith, O. 1844. Le vicaire de Wakefield. Paris : Hetzel. [1766].

Hamon, P. 1996. L'ironie littéraire. Essai sur les formes de l'écriture oblique. Paris: Hachette.

Iser, W. 1985. L'acte de lecture : théorie de l'effet esthétique. Bruxelles : Mardaga. [1972].

Jouve, V. 2001. "Qui parle dans le récit ? " Cahiers de narratologie 10-2 : 75-90.

Kayser, W. 1970, 1977. "Qui raconte le roman? ». Dans Poétique du récit, sous la direction de G. Genette et T. Todorov. Paris : Seuil : 59-84. [1958].

Langevin, F. 2011. "La posture exotique du narrateur-personnage. Inconfort et non-fiabilité dans quelques romans contemporains». Dans La transmission narrative. Modalités du pacte romanesque contemporain, sous la direction de F. Fortier et A. Mercier. Québec: Nota Bene : 207-233.

Lintvelt, J. 1989. Essai de typologie narrative. Le "point de vue». Théorie et analyse. Paris: Corti. [1981].

Maingueneau, M. 2004. Le discours littéraire. Paratopie et scène d'énonciation. Paris : Armand Colin.

Martinez-Bonati, F. 1980. “The Act of Writing Fiction.” New Literary History 11 : 425-434.

\section{Arborescences}

Revue d'études françaises

ISSN: 1925-5357 
Martinez-Bonati, F. 1981. Fictive Discourse and the Structure of Literature: A Phenomenological Approach. Ithaca : Cornell University Press.

Mercier, A. et F. Fortier. 2011. "La narration impossible. Conventions réalistes, catégories narratologiques et enjeux esthétiques ". Dans La transmission narrative. Modalités du pacte romanesque contemporain, sous la direction de F. Fortier et A. Mercier. Québec: Nota Bene : 333-356.

Nabokov, V. 1959. Lolita. Paris : Gallimard. [1955].

Nabokov, V. 1965. Feu pâle. Paris : Gallimard. [1962].

Nünning, A. F. 1999. "Unreliable, Compared to What? Towards a Cognitive Theory of Unreliable Narration: Prolegomena and Hypothesis." Dans Grenzüberschreitungen: Narratologie in Kontext / Transcending Boundaries: Narratology in Context, sous la direction de W. Grünzweig et A. Solbach. Tübingen : Gunter Narr Verlag : 53-73.

Nünning, A. F. 2005. "Reconceptualizing Unreliable Narration: Synthesizing Cognitive and Rhetorical Approaches." Dans A Companion to Narrative Theory, sous la direction de J. Phelan et P. Rabinowitz. Malden, Oxford et Victoria : Blackwell Publishing : 89-107.

Nünning, V. 2004. "Unreliable Narration and the Historical Variability of Values and Norms: The Vicar of Wakefield as a Test-case of a Cultural-Historical Narratology." Style XXXVIII-2 : 236-252. [1998].

Oléron, A. 2014. Le narrateur non fiable en question(s), parcours textuels et liberté du lecteur. Thèse de Doctorat en cours de réalisation, sous la direction de V. Jouve et F. Wagner. Université de Reims.

Olson, G. 2003. "Reconsidering Unreliability: Faillible and Untrustworthy Narrators." Narrative XI-1 : 93-109.

Patron, S. 2009. Le narrateur. Introduction à la théorie narrative. Paris : Armand Colin.

Perec, G. 1979. Un cabinet d'amateur. Histoire d'un tableau. Paris : Balland.

Phelan, J. 2005. Living to Tell About it: A Rhetoric and Ethics of Character Narration. Ithaca, New York et Londres : Cornell University Press.

Phelan, J. et P. Rabinowitz, dir. 2005. A Companion to Narrative Theory. Malden, Oxford et Victoria : Blackwell Publishing.

Rabatel, A. 2009. Homo narrans. Pour une analyse énonciative et interactionnelle du récit. Limoges : Lambert-Lucas.

Riggan, W. 1981. Picaros, Madmen, Naifs, and Clowns: The Unreliable First-Person Narrator. Norman : Oklahoma University Press.

Robbe-Grillet, A. 1955. Le voyeur. Paris : Minuit.

Robbe-Grillet, A. 2001. La reprise. Paris : Minuit.

Sarraute, N. 1956. L'ère du soupçon. Paris : Gallimard.

Toussaint, J.-P. 1997. La télévision. Paris : Minuit.

Viel, T. 1998. Le Black Note. Paris : Minuit.

Viel, T. 1999. Cinéma. Paris : Minuit.

Villiers de L'Isle-Adam, A. 1893. "À s'y méprendre ». Dans Contes cruels. Paris : CalmannLévy : 132-136.

Volodine, A. 1998. Le post-exotisme en dix leçons, leçon onze. Paris : Gallimard.

Volodine, A. 2002. Dondog. Paris : Seuil.

Wagner, F. 2003. «Instances lectrices et lecteur(s) réel(s)». La lecture littéraire 2003 : 333-353.

Wagner, F. 2011. "Retours, tours et détours du récit. Aspects de la transmission narrative dans quelques romans français contemporains ". Poétique $165: 3-20$.

Wagner, F. 2014. "Les voix du post-exotisme (sont-elles impénétrables ?) "Vox-poetica. http//www.vox-poetica.org/. Page consultée le 11 décembre 2015.

Arborescences

Revue d'études françaises

ISSN: 1925-5357 
Yacobi, T. 1981. "Fictional Reliability as a Communicative Problem." Poetics Today II-2 : 113126.

Yacobi, T. 2001. "Package Deals in Fictional Narrative: The Case of the Narrator's (Un)Reliability." Narrative IX-2 : 223-229.

Arborescences

Revue d'études françaises 